\title{
Research on the Effect of Hyperbaric Oxygen on Exercise-induced Fatigue in Rats
}

 \\ 1College of physical education, Jiangxi Science \& Technology Normal University, Nanchang \\ 330013, China \\ 2College of life science, Jiangxi Science \& Technology Normal University, Nanchang 330013, \\ China \\ achenyh@jxstnu.edu.cn, ${ }^{\mathrm{b}}$ liguoyin@jxstnu.edu.cn, ${ }^{\mathrm{c}} \mathrm{yuanch@jxstnu.edu.cn}$
}

Keywords: rats, hyperbaric oxygen, exercise-induced fatigue

\begin{abstract}
Hyperbaric oxygen therapy might be used to provide a means of therapy to help a speedier resumption to exercise-induced fatigue. In this study, forty SD rats were selected and randomly divided into four groups: control group, exercise-induced fatigue model group (fatigue group), hyperbaric oxygen therapy group (hyperoxia group) and hyperbaric oxygen pretreatment group before exercise (intervention group). The blood physiological and biochemical traits of the experiment rats were investigated. The results indicated that compared to the control group, the rats in fatigue group grew significantly slow $(P<0.01)$, while the intervention group and hyperoxia group had no significant difference in body weight, and compared with the control group, the white cell count, red blood cells count, hemoglobin, total superoxide dismutase (SOD) content of fatigue group, hyperoxia group and the intervention group decreased significantly $(P<0.01)$, and compared with fatigue group, the mean corpuscular volume, mean corpuscular hemoglobin content, SOD content of hyperoxia group and intervention group showed an increasing trend, while red blood cell count, hemoglobin and platelet count had a downward trend. It concluded that the hyperbaric oxygen could help to relieve fatigue and protect the growth and development of body and protect blood cells from the damage of exercise-induced fatigue, and improve immunity.
\end{abstract}

\section{Introduction}

With the rapid development of modern competitive sports, exercise-induced fatigue or disorder of athletes are growing severely. After exercise-induced fatigue, normal relaxation methods, pharmaceutical and other medical biology techniques and so on would be used to recover fatigue. In recent years medical hyperbaric oxygen therapy has been used in exercise-induced fatigue, and achieved good results[1,2,3,4]. Although hyperbaric oxygen had a good effect on exercise-induced fatigue, and there were some reports that mainly introduced the treatment effect of hyperbaric oxygen on exercise-induced fatigue, the mechanism of hyperbaric oxygen resistant to exercise-induced fatigue have not been reported. In order to make better use of hyperbaric oxygen to therapy exercise-induced fatigue, it is necessary to provide deep insight into the mechanisms of hyperbaric oxygen resistant to exercise-induced fatigue.

\section{Material and Methods}

In this experiment, forty SD rats were selected and randomly divided into four groups: control group, exercise-induced fatigue model group (fatigue group), hyperbaric oxygen therapy group 
(hyperoxia group) and hyperbaric oxygen pretreatment group before exercise (intervention group). Each group had 10 rats. The animal model of exercise-induced fatigue was established according to Bedford's. Standard medical hyperbaric oxygen treatment was used on the hyperoxia group and intervention group. Then blood samples were collected from four groups of experimental rats. We used automated hematology analyzer to test the blood physiological and biochemical traits, including white blood cell count, red blood cell count, red blood cell volume, the content of total hemoglobin, mean corpuscular hemoglobin platelet concentration, mean platelet volume, etc.. The experimental data were collected, and analyzed with SPSS software.

\section{Results and Discussion}

Before the experiment, all groups of the rats showed a good appetite, normal growth and sensitive action. After 28-day experiment, compared to the control group, the rats in fatigue group grew significantly slow $(\mathrm{P}<0.01)($ Fig.1), owed loss of appetite, malaise, lethargy and other symptoms of fatigue, while the intervention group and hyperoxia group had no significant difference in body weight, appetite, mental condition, physical fitness and so on. The mechanism that sports fatigue had an obvious effect on the growth and development of rats, might be that with the increasing exercise-fatigue, the harmful materials such as the oxygen free radicals appearing in rats harmed the cells, made the internal environment disorder and the energy consume[5].These adverse factors could cause a large number of cell apoptosis, on the other hand, those factors might seriously affect a series of physiological and biochemical reactions of body, which could result in the body's growth and development.

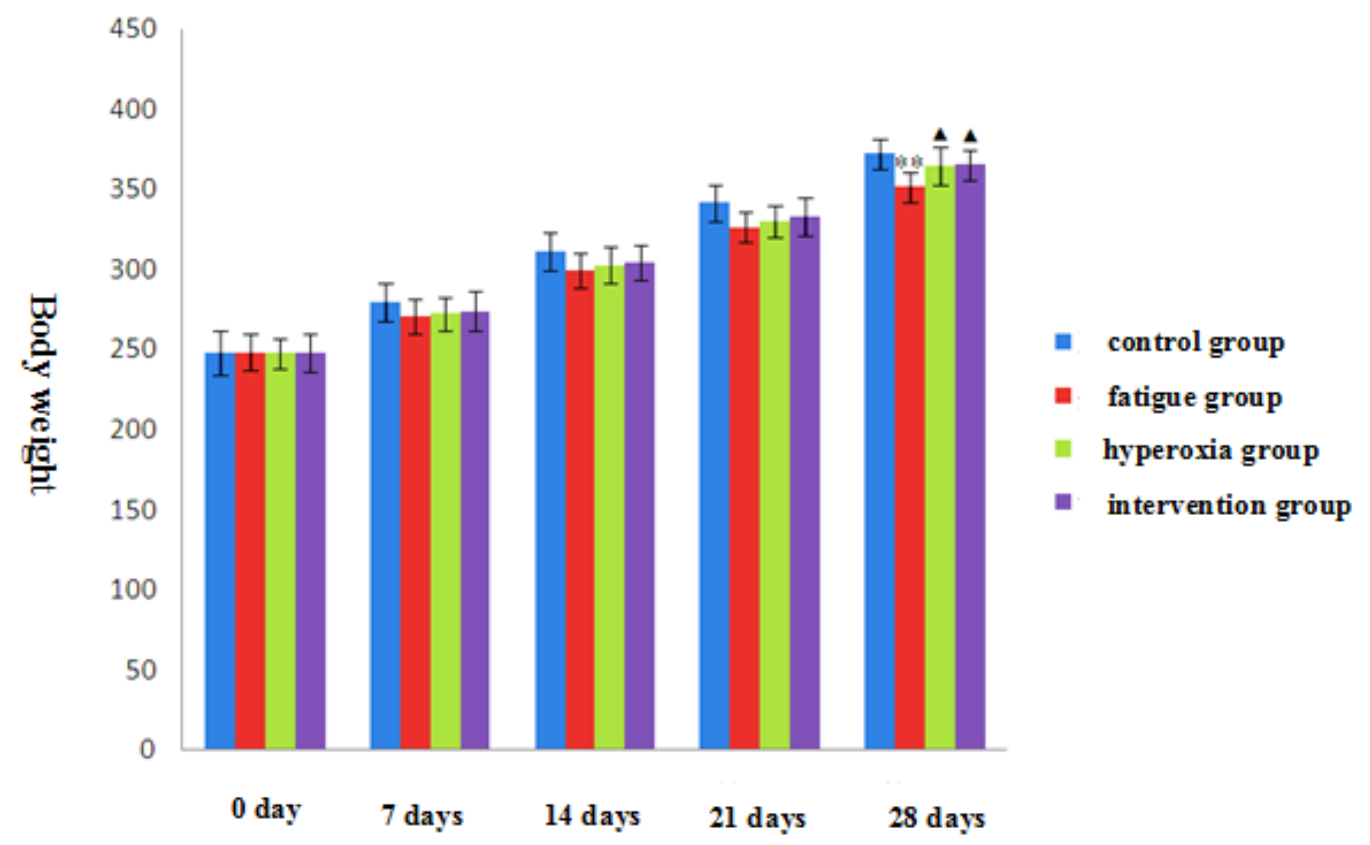

Fig.1 the effect of hyperbaric oxygen on the growth of rats

Note:compared with control group, ${ }^{*} \mathrm{P}<0.05,{ }^{*} \mathrm{P}<0.01$; compared with fatigue group, ${ }^{\boldsymbol{\Delta}} \mathrm{P}<0.05,{ }^{\boldsymbol{\Delta}}{ }^{\mathbf{P}}<0.01$.

Blood plays an important role in body metabolism and disease resistance. In this study, We used automated hematology analyzer to test the blood physiological and biochemical traits. The results, summarized in Table 1, indicated that compared with the control group, the white cell count, red blood cells count, hemoglobin, total superoxide dismutase (SOD) content of fatigue group, hyperoxia group and the intervention group were decreased significantly $(\mathrm{P}<0.01)$, while the mean corpuscular volume, average cell hemoglobin, platelet count showed an increasing trend. The reason that blood cells reduced might be that exercise-induced fatigue would make oxygen free 
radicals and other harmful metabolites increase, which would cause blood cell oxidative stress. And the oxidative stress would result in apoptosis which would lead to the decline in the number of blood cells $[6,7,8]$.

Table 1 The effect of hyperbaric oxygen on blood cells of rats

\begin{tabular}{|c|c|c|c|c|}
\hline Traits & Control group & Fatigue group & Hyperoxia group & Intervention group \\
\hline $\begin{array}{l}\text { White blood cell } \\
\text { count }\left(* 10^{9} / \mathrm{L}\right)\end{array}$ & $9.40 \pm 0.99$ & $4.66 \pm 0.95 * *$ & $5.62 \pm 1.12 * *$ & $6.23 \pm 0.74^{*} \boldsymbol{\Lambda}$ \\
\hline $\begin{array}{l}\text { Red blood cell } \\
\text { count }\left(* 10^{12} / \mathrm{L}\right)\end{array}$ & $9.63 \pm 0.27$ & $7.81 \pm 0.59 * *$ & $6.69 \pm 0.71 * * \Delta$ & $6.93 \pm 0.45^{* *} \boldsymbol{\Lambda}$ \\
\hline $\begin{array}{l}\text { Hematocrit } \\
(100 \%)\end{array}$ & $57.30 \pm 0.95$ & $49.33 \pm 2.86^{* *}$ & $44.35 \pm 3.70^{* * \boldsymbol{\Lambda}}$ & $43.88 \pm 4.22^{* *} \boldsymbol{\Lambda}$ \\
\hline $\begin{array}{l}\text { Mean corpuscular } \\
\text { volume (fl) }\end{array}$ & $59.53 \pm 0.67$ & $63.30 \pm 2.12 *$ & $66.56 \pm 2.40 * * \boldsymbol{\Lambda}$ & $63.27 \pm 2.10^{*}$ \\
\hline $\begin{array}{l}\text { Red cell } \\
\text { distribution } \quad \text { width } \\
(100 \%)\end{array}$ & $16.15 \pm 0.21$ & $14.56 \pm 0.63 *$ & $14.59 \pm 0.62 * *$ & $14.60 \pm 0.55^{*}$ \\
\hline Hemoglobin (g/l) & $167.67 \pm 4.16$ & $147.29 \pm 8.28 * *$ & $132.00 \pm 11.69^{* *} \mathbf{\Lambda}$ & $131.67 \pm 10.19 * * \boldsymbol{\Lambda}$ \\
\hline $\begin{array}{l}\text { Mean corpuscular } \\
\text { hemoglobin (pg) }\end{array}$ & $17.33 \pm 0.12$ & $18.81 \pm 0.52 * *$ & $19.73 \pm 0.49^{* * \Delta \mathbf{\Lambda}}$ & $18.95 \pm 0.33 * *$ \\
\hline $\begin{array}{l}\text { Platelet } \\
\left(* 10^{9} / \mathrm{L}\right)\end{array}$ & $516.50 \pm 17.68$ & $947.29 \pm 120.08 * *$ & $886.50 \pm 131.39 * *$ & $709.00 \pm 150.78^{\mathbf{\Lambda}}$ \\
\hline $\begin{array}{l}\text { Mean platelet } \\
\text { volume ( } \mathrm{fl} \text { ) }\end{array}$ & $11.27 \pm 0.91$ & $10.56 \pm 0.78$ & $11.63 \pm 1.71$ & $13.30 \pm 1.24 * \boldsymbol{\Lambda}$ \\
\hline $\begin{array}{l}\text { Platelet } \\
\text { distribution width } \\
(\%)\end{array}$ & $15.65 \pm 0.21$ & $15.43 \pm 0.18$ & $15.34 \pm 0.33$ & $15.58 \pm 0.48$ \\
\hline SOD（u/ml） & $591.50 \pm 64.35$ & $97.50 \pm 12.74 * *$ & $136.25 \pm 31.17 * * \mathbf{\Lambda}$ & $198.58 \pm 75.89 * * \boldsymbol{\Lambda \Lambda}$ \\
\hline
\end{tabular}

Compared with fatigue group, the mean corpuscular volume, mean corpuscular hemoglobin content, SOD content of hyperoxia group and intervention group showed an increasing trend, while red blood cell count, hemoglobin and platelet count had a downward trend. The reason that SOD content of hyperoxia group and intervention group rise might be that hyperbaric oxygen could clear up the oxygen free radicals, which might protect the cell from oxidative damage and avoid apoptosis, which would maintain the number of blood cells. So hyperbaric oxygen might relieve the body's fatigue by protecting the cells from damage.

\section{Conclusion}

Exercise-induced fatigue had a significant infaust effect on the growth and development of body, and the hyperbaric oxygen could help to relieve fatigue and protect the growth and development of body. Exercise-induced fatigue would reduce blood cell count, leading to immunity decreasing, and hyperbaric oxygen can protect blood cells from the damage of exercise-induced fatigue, and improve immunity.

\section{Acknowledge}


This study was supported by Research Project of Jiangxi Science \& Technology Normal University and Natural Science Foundation of Jiangxi Province.

\section{References}

[1] J. Stewart, D. Gosine, M. Kaleel and A. Kurtev: Unders Hyperb Med Vol.38(2011), p. 483-491

[2] Y. Ishii , M. Deie, and N. Adachi: Spo Med Vol.35(2005), p.739-746

[3] B.J. Kudchodkar , A. Pierce and L. Dory: Atherosclerosis Vol.193(2007), p.28-35.

[4] C.A. Godman, K.P. Chheda and L.E. Hightower: Cell stre \& chaper Vol.15(2010), p.431-442

[5] N.H. Secher, B. Quistorff and M.K. Dalsgaard: ugeskr Laeger Vol.168(2006), p.4503-4506

[6] N.Traiperm, H. Gatterer, M. Burtscher: Med Sci Spo Exer Vol.45(2013), p.1182-1187

[7] C.L.Wells, J.R. Stern and L.H. Hecht:Euro J App Phy Occu Phy Vol.48(1982),p.41-49

[8] E. Hessel, A. Haberland, M. Müller, D. Lerche and I. Schimke: Clin Chim Vol. 298 (2000), p.145-156 